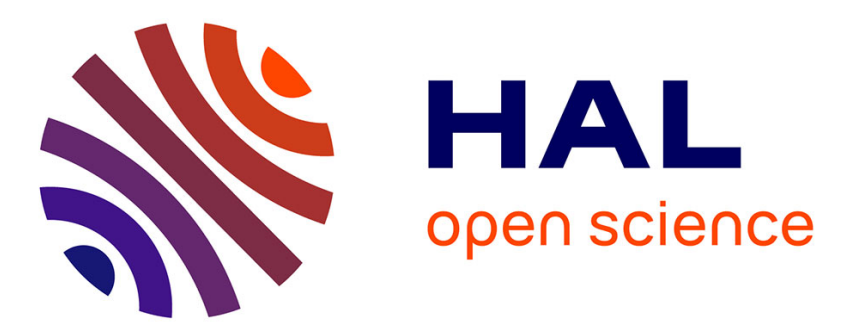

\title{
Programme orbite: trajectoires de particules dans un champ magnétique
}

\author{
$\mathrm{Ph}$. Leconte, J. Mougey
}

\section{To cite this version:}

Ph. Leconte, J. Mougey. Programme orbite: trajectoires de particules dans un champ magnétique. Revue de Physique Appliquée, 1969, 4 (2), pp.213-213. 10.1051/rphysap:0196900402021300 . jpa00243224

\section{HAL Id: jpa-00243224 https://hal.science/jpa-00243224}

Submitted on 1 Jan 1969

HAL is a multi-disciplinary open access archive for the deposit and dissemination of scientific research documents, whether they are published or not. The documents may come from teaching and research institutions in France or abroad, or from public or private research centers.
L'archive ouverte pluridisciplinaire HAL, est destinée au dépôt et à la diffusion de documents scientifiques de niveau recherche, publiés ou non, émanant des établissements d'enseignement et de recherche français ou étrangers, des laboratoires publics ou privés. 


\title{
PROGRAMME ORBITE : TRAJEGTOIRES DE PARTIGULES DANS UN GHAMP MAGNÉTIQUE
}

\author{
Ph. LEGONTE et J. MOUGEY, \\ Service de Physique Nucléaire à Haute Énergie, C.E.N., Saclay.
}

\begin{abstract}
Résumé. - Nous décrivons le programme Orbite qui calcule les trajectoires de particules chargées dans un champ magnétique.

Abstract. - We describe the Orbite code for calculating trajectories of charged particles in a magnetic field.
\end{abstract}

Les calculs d'optique des faisceaux de particules chargées dans des champs magnétiques peuvent être effectués de façon très simple par la méthode « matricielle »[1-2]. Cette méthode, qui consiste à repérer chaque trajectoire par rapport à une trajectoire de référence, ne se justifie plus dès que l'ensemble des trajectoires ne sont plus groupées, comme c'est le cas dans des spectromètres à grand angle solide, ou à grande acceptance en énergie. Nous avons été amenés, pour l'étude des spectromètres « 600 » et « 900 », à écrire un programme de calcul direct des trajectoires, qui peut permettre d'une part de déterminer les paramètres de la configuration du champ pour établir le projet d'un spectromètre, d'autre part d'étalonner un aimant donné dont on a mesuré la carte du champ.

Le programme Orbite ayant été conçu pour des calculs de spectromètres à fer, la configuration du champ dans la zone traversée par les particules est supposée avoir un plan de symétrie, dont les trajectoires s'écartent peu. Le champ est donné uniquement dans ce plan de symétrie. Il est calculé, hors du plan, en tenant compte du fait que l'on a $\operatorname{rot} \mathbf{B}=\operatorname{div} \mathbf{B}=0$ et par des développements limités aux termes en $z^{2}$. La carte de champ est divisée en trois zones : i) une zone « centrale », dans laquelle le champ est défini en coordonnées cylindriques, soit par une fonction analytique $B(r, \theta, 0)$ (variante Orbite 1), soit par la valeur du champ en tous les points d'un maillage $(r, \theta)$ (variante Orbite 2); ii) deux zones de champs « de bord », où la valeur du champ est définie en tous points d'un maillage en coordonnées cartésiennes. Le champ en un point quelconque d'une maille est obtenu par interpolation au deuxième ordre.

L'intégration de l'équation du mouvement

$$
m \boldsymbol{\gamma}=\mathrm{ev} \Lambda \mathbf{B}
$$

s'effectue pas à pas en admettant que, dans l'intervalle considéré, le champ est homogène, ce qui entraîne que l'élément de trajectoire est un arc d'hélice. Le point « image » peut être calculé pour un ensemble de trajectoires de même impulsion, comme correspondant au minimum de l'enveloppe de ce faisceau de trajectoires. Le plan focal de l'aimant peut ainsi être défini comme étant le lieu de ce minimum, en fonction de l'impulsion.

La sortie des résultats peut se faire selon plusieurs options, notamment sous forme de figures sur traceur Benson, représentant $: i)$ soit les trajectoires projetées sur le plan de symétrie dans la zone image, ou à travers tout l'aimant; ii) soit la section d'un faisceau de trajectoires perpendiculairement à la trajectoire de référence. Il est possible de suivre chaque trajectoire à travers les champs magnétiques de plusieurs aimants successifs, ce qui permet l'utilisation du programme Orbite pour l'étalonnage d'un système de transport de faisceaux. Chaque aimant est repéré par rapport à l'aimant qui le précède, ce qui permet également d'étudier l'effet d'un mauvais alignement.

Le programme est actuellement disponible sur IBM 360-75. A titre d'exemple, le calcul de 45 trajectoires, comptant chacune environ 4000 pas d'intégration, s'effectue en 5,5 mn.

\section{BIBLIOGRAPHIE}

[1] Pennner (S.), Rev. Sci. Instr., 1961, 32, 150.

[2] Brown (K. L.), SLAC report 75. 